\title{
Soluble urokinase plasminogen activator receptor is linearly associated with dietary quality and predicts mortality
}

\author{
Peter B. S. Törnkvist ${ }^{1}$, Thomas H. Haupt ${ }^{1}$, Line J. H. Rasmussen ${ }^{1}$, Steen Ladelund ${ }^{1}$, Ulla Toft $^{2}$, \\ Charlotta Pisinger ${ }^{2}$ and Jesper Eugen-Olsen ${ }^{1}$ \\ ${ }^{1}$ Clinical Research Centre, Copenhagen University Hospital Hvidovre, Kettegård Allé 30, 2650 Hvidovre, Denmark \\ ${ }^{2}$ Research Centre for Clinical Research and Prevention, Frederiksberg Hospital, Hovedvejen, Entrance 5, Nordre Fasanvej 57, \\ 2000 Frederiksberg, Denmark
}

(Submitted 6 November 2017 - Final revision received 31 October 2018 - Accepted 7 December 2018 - First published online 7 February 2019)

\section{Abstract}

Chronic inflammation is associated with disease risk and mortality in the general population. Soluble urokinase plasminogen activator receptor (suPAR) is a stable marker of chronic inflammation, and a higher serum-concentration of suPAR is found in individuals with an unhealthy lifestyle such as smoking. This article investigates the association between suPAR and dietary quality measured with the dietary quality score (DQS). The DQS is an index of the overall quality of an individual's dietary habits assessed through a self-administered FFQ. Furthermore, this article investigates the association of both suPAR and the DQS with CVD risk and mortality in the general Danish population. We analysed 5347 individuals aged 30-60 years from the Danish Inter99 study cohort. Multiple linear regression analyses showed a linear inverse association between the DQS and suPAR $(P=0.0005)$. Cox regression analyses showed an $18(95 \%$ CI 9,26$) \%$ increase in the risk of death from any cause with each $1 \mathrm{ng} / \mathrm{ml}$ increase in suPAR. We found no significant association between the DQS and the mortality (hazard ratio: $1 \cdot 16,95 \%$ CI $0.79,1.69)$. All analyses were adjusted for demographics and lifestyle factors. The association between the DQS and suPAR on the one hand and suPAR and mortality on the other supports the argument that low dietary quality may constitute a health risk through its influence on chronic inflammation. Future research should examine whether suPAR is modifiable through changes in dietary habits.

Key words: Inflammation: Dietary quality: Soluble urokinase plasminogen activator receptor: Biomarkers: Epidemiological studies

Systemic low-grade (or chronic) inflammation is widely recognised as a modifiable risk factor for disease and premature death $^{(1,2)}$. Knowledge about how lifestyle affects the inflammatory status is therefore of importance for health professionals if they are to reduce the risk of disease.

Soluble urokinase plasminogen activator receptor (suPAR) has been established as a stable marker of chronic inflammation and as a predictor of disease and mortality in the general population ${ }^{(3-5)}$. suPAR has also been shown to predict disease severity and mortality in acutely admitted medical patients ${ }^{(6,7)}$. It has previously been shown that the serum SUPAR concentration in the general population is associated with lifestyle habits including alcohol (U shaped) and diet (linear) ${ }^{(8,9)}$. The relationship between diet and inflammation is supported by several studies that show associations between healthy dietary patterns and lower serum concentrations of high-sensitivity C-reactive protein and IL- $6^{(10-13)}$. Dietary habits are an important risk factor for CVD and overall mortality ${ }^{(14-18)}$. This relates to dietary components as well as overall dietary quality. A healthy diet is recognised as a diet high in fruits, vegetables, fish and fibre and low in saturated animal fat. About a decade ago, it was noted that the study of dietary patterns is important because such patterns express the complex nature of a composite diet and the actions and interactions of the diet in the body more accurately than the study of single nutrients is able to ${ }^{(18)}$.

Though a relationship between overall dietary quality and suPAR has been shown earlier ${ }^{(9)}$, a specific pattern for this relationship has not been established. The aim of this study was to investigate the relationship between dietary quality and suPAR in detail as well as to investigate the association of each of these factors with mortality and future IHD and stroke. This investigation was carried out using a large random sample of the general Danish population followed for 12 years.

\section{Methods}

\section{Study population}

This study includes participants from the Inter99 study. Inter99 was a randomised intervention study investigating the effect of

Abbreviations: DQS, dietary quality score, suPAR, soluble urokinase plasminogen activator receptor.

* Corresponding author: P. B. S. Törnkvist, email pt.forsk@posteo.dk 


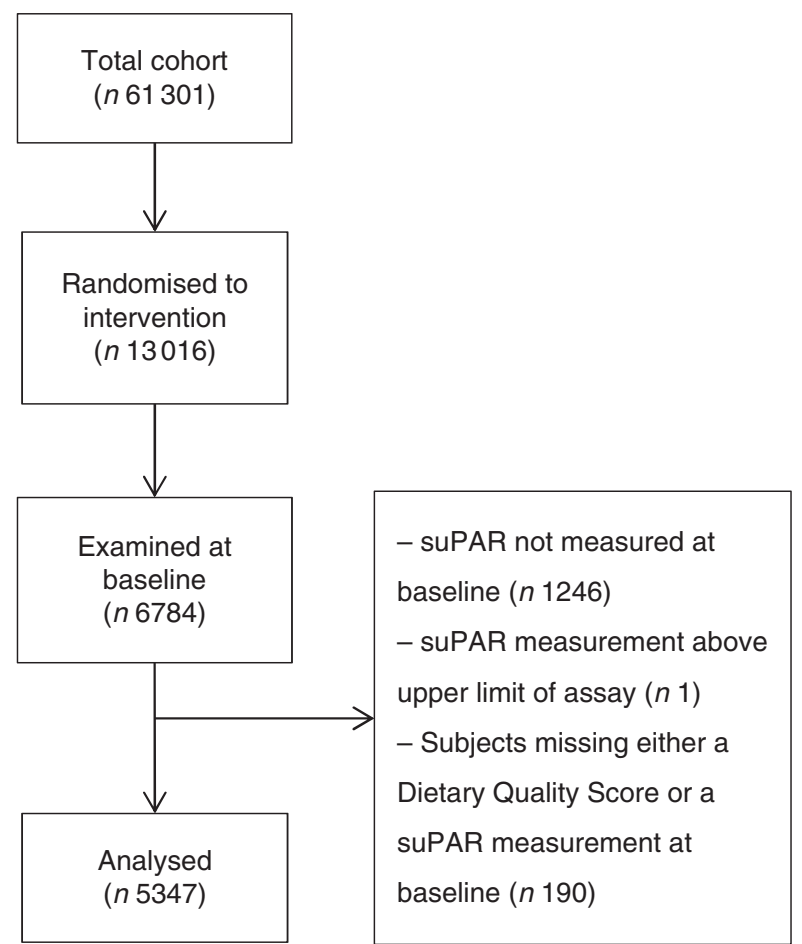

Fig. 1. Flowchart of study population. suPAR, soluble urokinase plasminogen activator receptor.

individual lifestyle counselling and group-based lifestyle education on the risk of CVD. Detailed design and results are presented elsewhere ${ }^{(19,20)}$. In short, the Inter99 cohort comprised 61301 randomly selected individuals aged 30-60 years from the southwestern area of the Capital Region of Denmark. Of these, $13016(21.2 \%)$ were randomly assigned to the intervention group and $6784(52 \cdot 1 \%)$ were examined at baseline in 1999-2001. We analysed the 5347 (78.8\%) of the examined participants who had both a suPAR measurement and a dietary quality score (DQS) available (Fig. 1). Characteristics of excluded participants are shown in online Supplementary Tables S1 and S2.

\section{Data collection}

The collection of data is described in detail elsewhere ${ }^{(19,20)}$. In short, the participants answered a detailed questionnaire about demographics, lifestyle habits and healthcare contacts and underwent a physical examination at baseline. Fasting blood samples were drawn from all participants. Total cholesterol was measured instantly and the blood samples were stored at $-80^{\circ} \mathrm{C}$ for later analysis of the remaining biomarkers ${ }^{(19,20)}$. Efficient matching of collected data with the individual study participant was achieved through linking of the data to personal id numbers (Central Person Registry (CPR)-numbers) ${ }^{(21)}$.

Demographics. Data on sex and age were extracted from the Danish Civil Registration System using each participant's CPR number. Socio-economic status was stratified into three groups based on the length of vocational training/tertiary education: $<2$ years ('low'), 2-4 years ('average') and >4 years ('high').
Lifestyle habits. Lifestyle habits were self-reported by questionnaire. Dietary intake was measured using a validated selfadministered forty-eight-item FFQ including questions about the intake of fruit, vegetables, fish and fat. From this, the DQS was calculated. The DQS was developed on the basis of Danish national dietary guidelines in 2007 by Toft et al. ${ }^{(22)}$ using a forty-eight-item FFQ and validated against a 198-item FFQ. The DQS divides individuals into one of nine categories based on questions regarding the intake of fruits, boiled vegetables, raw vegetables, vegetable or vegetarian dishes, fish for lunch and fish for dinner, fat as spread and fat used at preparation. The categories of the DQS range from non-prudent (group 1) to prudent (group 9) dietary habits ${ }^{(22)}$. For fruit, vegetables and fish, the score was based on quantity; but for fat, points were assigned based on the type of fat. Baseline smoking habits were categorised as daily smoker (min. 1 cigarette/g tobacco per d), occasional smoker, former smoker or never smoker. Weekly alcohol consumption (in units of $12 \mathrm{~g}$ alcohol/week) was divided into four groups based on national health recommendations in 1999: abstinent (0 units/week), within recommendations (1-14 units/week for women, 1-21 units/week for men), borderline consumption (14-35 units/week for women, 21-35 units/week for men) and overuse (more than 35 units/ week for all). Physical activity was defined as the sum of leisure time physical activity and the amount of the time spent on transportation to and from work, consisting of walking, running or biking ${ }^{(23)}$. The sum was converted into minutes a week, using a 5 -d working week, and was divided into four groups: $0-112 \cdot 5,142 \cdot 5-225,255-420$ and 450-720 $\mathrm{min} /$ week.

Recording of endpoints. Data on endpoints were extracted from Danish registries using the participants' unique CPR number. Deaths were extracted from the Danish Civil Registration System ${ }^{(21)}$, hospital admissions from the National Patient Registry $^{(24)}$ and causes of death from the National Registry of Causes of Death ${ }^{(20,25)}$. IHD was defined as the International Classification of Diseases (ICD)-8 codes $410-414$ or ICD-10 codes I20-I25 or as relevant cardiac surgery (bypass, recanalisation or reconstruction of coronary arteries). Stroke included both haemorrhagic and ischaemic stroke and was defined as the ICD-8 codes $430-434$ or 436 or ICD-10 codes I60-I64 or I $69^{(20)}$

Biomarker measurements. The baseline plasma samples were collected in $1999-2001$ and stored at $-80^{\circ} \mathrm{C}$ until measurement of suPAR, which took place in 2011. suPAR levels were determined using the suPARnostic sandwich ELISA (ViroGates $\mathrm{A} / \mathrm{S})^{(9)}$. One participant with a suPAR concentration above $22 \mathrm{ng} / \mathrm{ml}$ (upper limit of assay) was excluded from further analyses. The concentration of total cholesterol, HDLcholesterol and TAG were determined on fresh unfrozen samples at baseline (1999-2001) by enzymatic techniques with the Hitachi 912 System (Roche Diagnostics) ${ }^{(19)}$. The concentration of LDL-cholesterol was calculated by Friedewald's equation ${ }^{(26)}$. Participants had their weight and height measured, and BMI was calculated as weight $(\mathrm{kg}) /$ height $(\mathrm{m})^{2(19)}$. 


\section{Ethics}

All participants provided written informed consent before taking part in the study. The study was approved by the local ethics committee (KA 98 155), by the Danish Data Protection Agency, and it was conducted in accordance with the Declaration of Helsinki. The study is registered with ClinicalTrials.gov (NCT00289237).

\section{Statistics}

Baseline characteristics were described by mean value, standard deviation and range for continuous variables and number of observations and percentages for categorical variables.

To guide the analysis plan, we created a directed acyclic graph (Fig. 2) ${ }^{(27)}$. The graph contains all investigated factors, and arrows indicate a causal relation. This visualisation allowed for a ready identification of potential confounders. The causal pattern and adjustments for confounders enables the control for unknown confounding by closing all 'backdoors' in the directed acyclic graph ${ }^{(27)}$. When assessing the total effect of diet on endpoints, we did not adjust for intermediary factors to avoid overadjustment biases and unnecessary adjustments ${ }^{(28)}$. From the directed acyclic graph, it is further visualised that adjusting for suPAR in an analysis of the dietary effect on endpoints provides an assessment of all effects of diet on endpoints that is not mediated by suPAR.

In the univariate analyses, we used one-way ANOVA to test the distributions of biomarkers (suPAR, total cholesterol, HDLcholesterol and BMI) in the groups of the DQS. We tested the distributions of these biomarkers in the individual food groups as well, if an association with the DQS was found. The adjusted analyses were multiple linear regressions with $\log _{2}$-transformed suPAR, total cholesterol, HDL-cholesterol and BMI modelled as functions of sex, age, lifestyle factors (smoking habits, alcohol intake and physical activity) and the DQS (reference was DQS = group 5). In the adjusted analyses, we allowed for an interaction between sex and age. In addition, the effect of the DQS on biomarkers was modelled as a continuous variable, as the DQS showed no departure from trend $(P=0 \cdot 27-0 \cdot 81)$. Estimates were back transformed by $\left(2^{\beta}-1\right) \times 100 \%$ and interpreted as percentage difference in biomarker concentration.

Data on endpoints are presented as mean follow-up time in years, number and percentage of participants who reached the specific endpoints, events per 1000 person years and, for IHD and stroke, the case fatality rates. We analysed time to IHD, stroke and death with cumulative incidence plots stratified by the DQS (dichotomised into "unhealthy diet' (scores 1-3) and 'intermediate/healthy diet' (scores 4-9)) and sex- and age-specific ( $\leq 45$ or $>45$ years) suPAR quartiles. For both IHD and stroke, death was set as a competing endpoint. Log-rank tests were carried out to determine whether the cumulative incidence was different between the groups. Furthermore, time-to-event were analysed by the Cox proportional hazards method to estimate three effects: any total effect of DQS, any effect of DQS not going through changes in suPAR concentration and any total effect of suPAR. Thus, three models were devised for each endpoint:

(1) The total dietary effect with dichotomised DQS, sex, age and lifestyle factors as explanatory variables.

(2) The suPAR-adjusted dietary effect with dichotomised DQS, sex, age, lifestyle factors and suPAR concentration as explanatory variables.

(3) The total suPAR effect with suPAR concentration, sex, age, dichotomised DQS, lifestyle factors, total and HDL-cholesterol, blood pressure, prevalent diabetes mellitus and prevalent CVD as explanatory variables.

For all survival analyses, participants with prior IHD was excluded from analyses with IHD as the endpoint. The same approach was used for stroke. All Cox models were performed

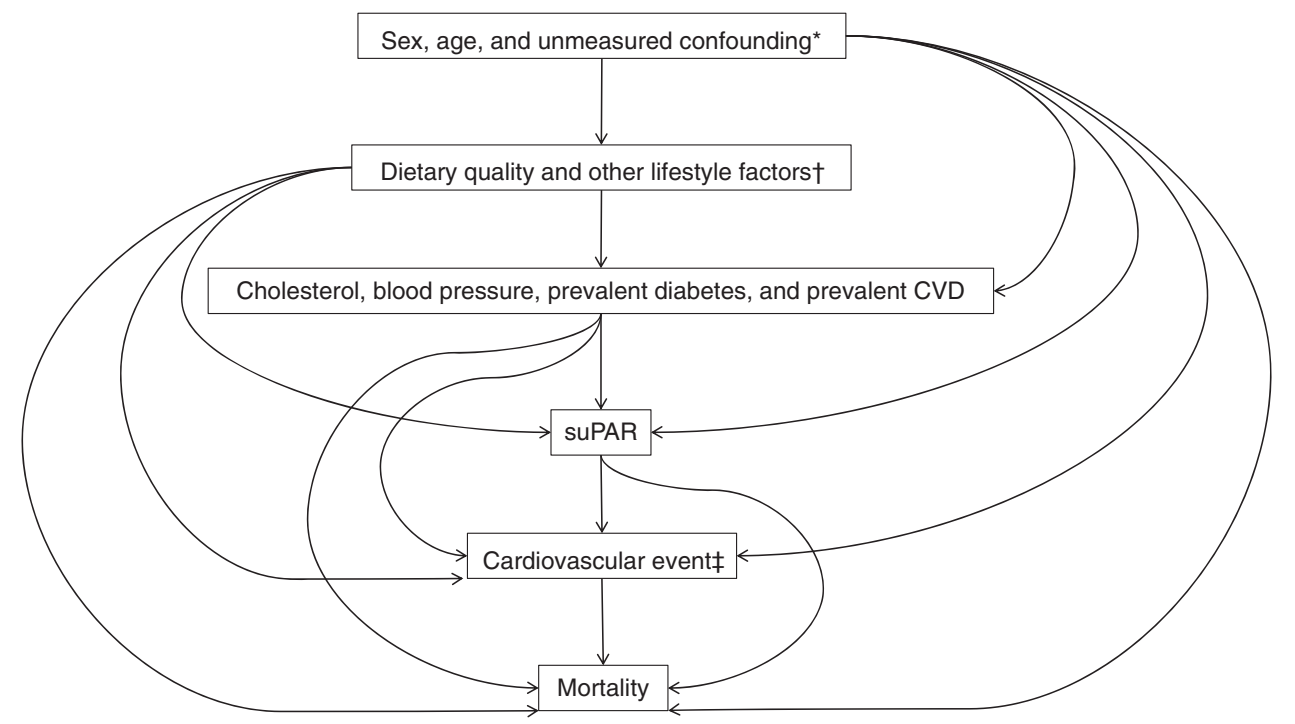

Fig. 2. Directed acyclic graph showing the hypothesised causality between the investigated factors and confounders. The causality relations of the factors are visualised by arrows. * Unmeasured confounders include genetic makeup and other known or unknown individual characteristics that affect behaviour and health. † Other lifestyle factors include smoking habits, alcohol consumption and physical activity. $\ddagger$ Cardiovascular event includes IHD and stroke. suPAR, soluble urokinase plasminogen activator receptor. 
as single failure-time analyses. Data were right censored at the end of follow-up, and for IHD and stroke we used a censoring variable with three levels: 0 (no event in the follow-up period), 1 (event within the follow-up period) and 2 (death within the follow-up period). For death, we used a censoring variable with two levels: 0 (no event in the follow-up period) and 1 (death within the follow-up period). Time to event was measured from the date of enrolment into the study (between March 1999 and January 2001), and follow-up in national registries was carried out until 11th of July 2011. Time on study in days was used as the underlying timescale. We tested the potential interaction between age and diet and age and suPAR, respectively; the estimates are shown in online Supplementary Table S3.

To quantify the effect of missing values on the regression results, we did the following modelling: we performed each regression twice for each confounding variable containing missing values (physical activity, alcohol consumption and smoking status). First, we performed the regression excluding the variable of interest. Then, we performed the same regression but excluding participants with a missing value in the variable of interest. Last, we compared the estimates from the two regressions.

R 3.2.3 (R Foundation) and SAS 9.4 (SAS Institute) were used for statistical analyses. In the Cox regressions, the Efron approximation was used to handle tied observations.

\section{Results}

\section{Cohort characteristics}

In total, 5347 participants $(51.5 \%$ women, $P=0.03)$ were included. Baseline characteristics are presented in Table 1.

\section{Soluble urokinase plasminogen activator receptor and dietary quality score}

When suPAR concentrations were stratified according to the DQS groups and tested with one-way ANOVA, we found a significant difference in the means of the groups (Table 2; $P<0.0001)$. When adjusted for sex, age and lifestyle factors (smoking habits, alcohol intake and physical activity) in the linear model, suPAR was associated with the DQS $\left(\beta_{\text {group } 1}=10.0 \quad(-1 \cdot 3-22.6) \% ; \beta_{\text {group } 9}=-2.9(-14 \cdot 8-10 \cdot 6) \%\right.$; reference $=$ group $5 ; P=0.0005$; Fig. 3). The linear effect of the DQS on suPAR was a $1.6 \%$ decrease (95\% CI $1.0,2.3 \%$, $P<0.0001)$ in suPAR per one step increase in DQS. When instead stratifying suPAR concentrations according to the food groups comprising the DQS (fruit, vegetables, fish and fat), we found a significantly different distribution of suPAR values between the healthy, intermediate and unhealthy group in each food group except in the 'fish' group (Table 3).

\section{Cholesterol, BMI and dietary quality score}

HDL-cholesterol was distributed significantly different between the groups of the DQS (Table 2; $P<0 \cdot 0001$ ). This was not the
Table 1. Characteristics of the study cohort at baseline (Numbers, percentages and ranges; mean values and standard deviations)

\begin{tabular}{|c|c|c|c|}
\hline Variable (unit) & $n$ & $\%$ & Range \\
\hline Age (years) & 5347 & & $29 \cdot 7-61 \cdot 3$ \\
\hline Mean & \multicolumn{2}{|c|}{$45 \cdot 9$} & \\
\hline SD & \multicolumn{2}{|c|}{$8 \cdot 0$} & \\
\hline Sex & 5347 & & \\
\hline Female & 2754 & $51 \cdot 5$ & \\
\hline Male & 2593 & $48 \cdot 5$ & \\
\hline suPAR (ng/ml) & 5347 & & $0.65-21 \cdot 2$ \\
\hline Mean & & & \\
\hline SD & & & \\
\hline Total cholesterol (mmol/l) & 5345 & & $2 \cdot 30-12 \cdot 2$ \\
\hline Mean & & & \\
\hline SD & & & \\
\hline HDL-cholesterol (mmol/l) & 5346 & & $0 \cdot 25-3 \cdot 71$ \\
\hline Mean & & & \\
\hline SD & & & \\
\hline LDL-cholesterol (mmol/l) & 5280 & & $0.80-7.90$ \\
\hline Mean & & & \\
\hline SD & & & \\
\hline TAG (mmol/l) & 5345 & & $0 \cdot 30-36 \cdot 0$ \\
\hline Mean & & & \\
\hline SD & & & \\
\hline Systolic blood pressure (mmHg) & 5346 & & $83-213$ \\
\hline Mean & & & \\
\hline SD & & & \\
\hline Diastolic blood pressure $(\mathrm{mmHg})$ & 5345 & & $30-140$ \\
\hline Mean & & & \\
\hline SD & & & \\
\hline BMI $\left(\mathrm{kg} / \mathrm{m}^{2}\right)$ & 5344 & & $14 \cdot 9-55 \cdot 7$ \\
\hline Mean & & & \\
\hline SD & & & \\
\hline Prevalent diabetes & 303 & $5 \cdot 7$ & \\
\hline Prevalent CVD & 99 & 1.9 & \\
\hline Smoking status* & 5347 & & \\
\hline Daily smoker & 1887 & $35 \cdot 3$ & \\
\hline Occasional smoker & 200 & 3.7 & \\
\hline Former smoker & 1341 & $25 \cdot 1$ & \\
\hline Never smoker & 1902 & 35.6 & \\
\hline Alcohol consumption* & 5347 & & \\
\hline Abstinent & 519 & $9 \cdot 7$ & \\
\hline Within recommendations & 3814 & 71.4 & \\
\hline Borderline & 608 & 11.4 & \\
\hline Overuse & 203 & $3 \cdot 8$ & \\
\hline Physical activity* & 5347 & & \\
\hline $0-112.5 \mathrm{~min}$ & 644 & $12 \cdot 0$ & \\
\hline $142 \cdot 5-225 \mathrm{~min}$ & 1129 & $21 \cdot 1$ & \\
\hline $255-420 \mathrm{~min}$ & 2630 & $49 \cdot 2$ & \\
\hline $450-720 \mathrm{~min}$ & 635 & 11.9 & \\
\hline Socio-economic status* & 5347 & & \\
\hline Low & 1090 & $20 \cdot 4$ & \\
\hline Medium & 3278 & $61 \cdot 3$ & \\
\hline High & 576 & $10 \cdot 8$ & \\
\hline
\end{tabular}

suPAR, soluble urokinase plasminogen activator receptor. ${ }^{*}$ Missing data are not shown.

case for total cholesterol (Table 2; $P=0 \cdot 10$ ) nor BMI (Table 2; $P=0 \cdot 27$ ). When adjusted for sex, age and lifestyle factors, borderline associations with the DQS were found for total cholesterol $\left(\beta_{\text {group } 1}=2.4(-3.7\right.$ to 8.8$) \% ; \beta_{\text {group } 9}=-6.5(-13.1$ to $0.7) \%$; reference $=$ group $5 ; \quad P=0.07 ;$ Fig. 3) and HDLcholesterol $\left(\beta_{\text {group } 1}=3.7(-4.4\right.$ to 12.5$) \% ; \beta_{\text {group } 9}=-0.3(-9 \cdot 6$ to 9.9$) \%$; reference $=$ group $5 ; P=0.07$; Fig. 3$)$. No association was found with BMI $\left(\beta_{\text {group } 1}=1.9(-3.4\right.$ to 7.5$) \% ; \beta_{\text {group } 9}=3.24$ $(-3.0$ to 10.3$) \%$; reference $=$ group $5 ; P=0 \cdot 16$; Fig. 3$)$. The linear 
suPAR is associated with dietary quality

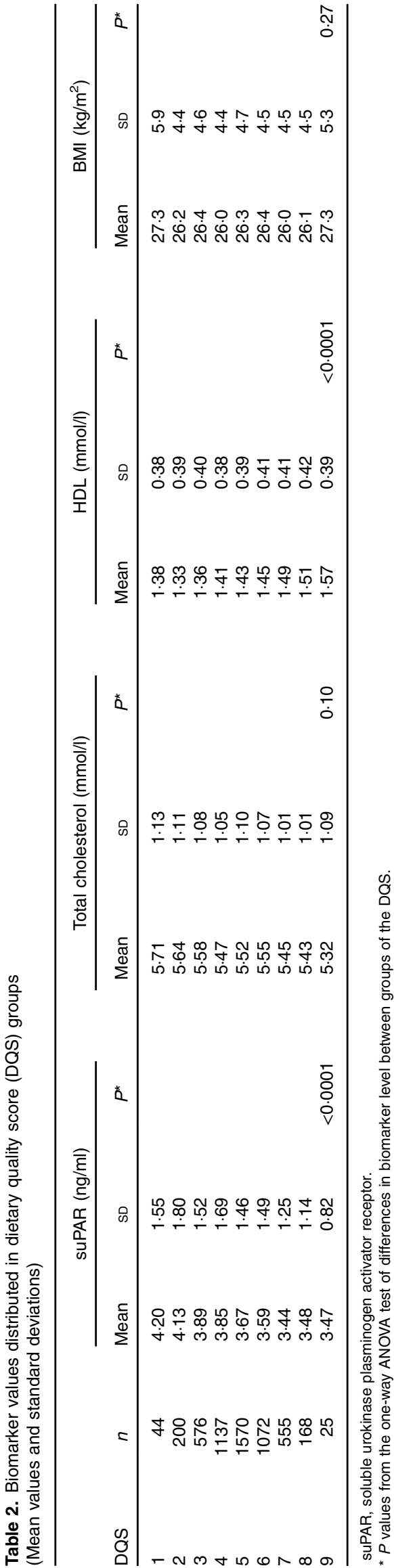

effect of the DQS was a $0.5 \%(95 \%$ CI $0.2,0.9 \%, P=0.007)$ decrease in total cholesterol and a $0.7 \%$ (95\% CI $0.2,1.2 \%$, $P=0.0065)$ decrease in HDL-cholesterol per one step increase in DQS. BMI showed no association with the continuous version of the DQS. As none of the cardiovascular risk markers were significantly associated with the categorical DQS in the adjusted model, we did not examine the univariate distributions of these markers in the food groups.

\section{Dietary quality score, soluble urokinase plasminogen} activator receptor and risk of IHD, stroke and death

During 11.3 years of mean follow-up, 339 (6.3\%) were diagnosed with IHD (5.6/1000 person-years, case fatality rate $=3.5 \%), 189$ (3.5\%) were diagnosed with stroke $(3 \cdot 1 / 1000$ person-years, case fatality rate $=2 \cdot 1 \%)$ and $215(4.0 \%)$ had died (3.6/1000 personyears). Fig. 4 shows cumulative incidence plots for IHD, stroke and death according to dichotomised DQS (unhealthy $v$. average/ healthy) and sex- and age-specific suPAR quartiles. The DQS was significantly associated with IHD and mortality but not with stroke (Fig. 4(a)-(c)), whereas suPAR was significantly associated with stroke and mortality (Fig. 4(e) and (f)) and borderline associated with IHD (Fig. 4(d)). When adjusted for sex, age and lifestyle factors, low dietary quality showed no significant association with IHD risk, stroke risk or mortality (Table 4). Similar results were found in the suPAR-adjusted model (Table 4). The total effect of suPAR was a $13(95 \%$ CI 2, 25) \% increase in the risk of stroke and an 18 (95\% CI 9, 26)\% increase in the risk of death from any cause for each $1 \mathrm{ng} / \mathrm{ml}$ increase in suPAR, while no association was found between suPAR and IHD risk (Table 4).

\section{Discussion}

In this large cohort study of randomly selected individuals from the general population, we showed that the serum concentration of the inflammatory biomarker suPAR was inversely associated with the DQS. When following the participants for 12 years, a higher suPAR concentration was associated with greater risk of stroke and higher mortality but not with IHD risk.

To compare suPAR to established biomarkers of CVD risk, we examined the association of the DQS with total and HDLcholesterol and BMI. None of these cardiovascular risk markers was associated with the DQS as a categorical variable adjusted for sex, age and lifestyle factors (smoking habits, alcohol intake and physical activity). When treated as a continuous variable, the DQS was associated with total and HDL-cholesterol, indicating that the dietary quality does affect plasma cholesterol concentration. Toft et $a l^{(22)}$ has investigated the association of the DQS with total and HDL-cholesterol and BMI in the same cohort and found a significant trend toward lower total cholesterol concentrations in subjects with higher DQS, but no association between the DQS and HDL-cholesterol or BMI. The difference between the findings by Toft et al. and our results may be due to the adjustment of alcohol intake and the smaller number of analysed participants in the present study. Both HDL-cholesterol and BMI are affected by intake of refined sugars $^{(29,30)}$, and since the DQS does not incorporate an 
(a)

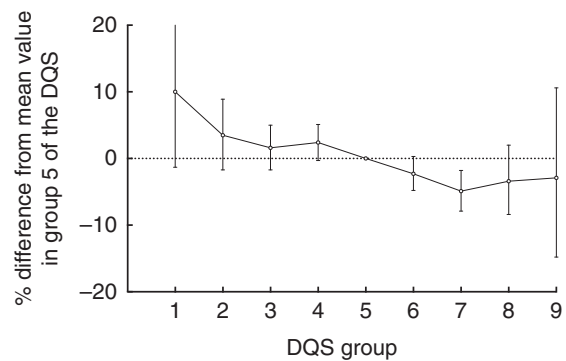

(c)

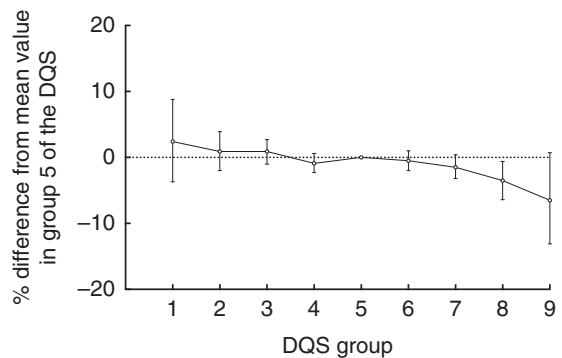

(b)

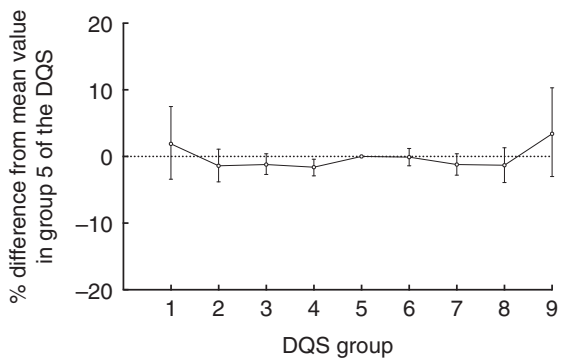

(d)

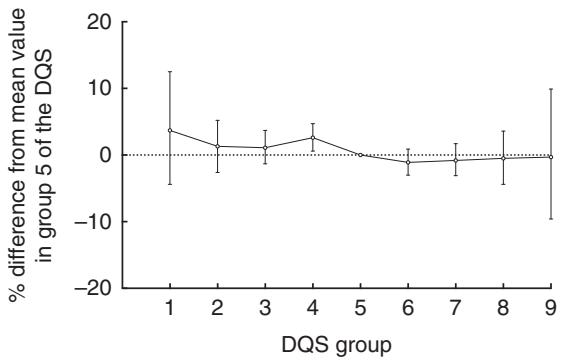

Fig. 3. Percentage differences in level of (a) soluble urokinase plasminogen activator receptor (suPAR), (b) BMI, (c) total cholesterol and (d) HDL-cholesterol between each group of the dietary quality score (DQS) and group 5 . Adjusted for sex, age and smoking habits; $95 \% \mathrm{Cl}$ indicated by error bars.

Table 3. Soluble urokinase plasminogen activator receptor (suPAR) values distributed in food component groups (Mean values and standard deviations)

\begin{tabular}{|c|c|c|c|c|}
\hline \multirow[b]{2}{*}{ Food component } & \multirow[b]{2}{*}{$n$} & \multicolumn{3}{|c|}{ suPAR (ng/ml) } \\
\hline & & Mean & SD & $P^{*}$ \\
\hline \multicolumn{5}{|l|}{ Fruit } \\
\hline Unhealthy & 1476 & 3.90 & 1.59 & \\
\hline Intermediate & 3214 & 3.66 & 1.53 & \\
\hline Healthy & 656 & 3.50 & 1.23 & $<0.0001$ \\
\hline \multicolumn{5}{|l|}{ Vegetables } \\
\hline Unhealthy & 911 & 3.92 & 1.53 & \\
\hline Intermediate & 3433 & 3.69 & 1.52 & \\
\hline Healthy & 1003 & 3.57 & 1.46 & $<0.0001$ \\
\hline \multicolumn{5}{|l|}{ Fish } \\
\hline Unhealthy & 1335 & 3.75 & 1.55 & \\
\hline Intermediate & 3226 & 3.71 & 1.55 & \\
\hline Healthy & 786 & 3.63 & 1.29 & 0.23 \\
\hline \multicolumn{5}{|l|}{ Fat } \\
\hline Unhealthy & 381 & 4.06 & 1.60 & \\
\hline Intermediate & 3586 & 3.75 & 1.59 & \\
\hline Healthy & 1380 & 3.51 & 1.23 & $<0.0001$ \\
\hline
\end{tabular}

* $P$ values from the one-way ANOVA test of differences in suPAR concentration between subgroups in each food component group.

estimation of refined sugar intake, we were unable to control for this effect. In addition, the DQS correlates with higher total energy intake ${ }^{(22)}$ but no unique estimation of this factor is available in the score. This made us unable to control for any effect of total energy intake on biomarker concentrations and to estimate the relative intake of the individual food groups comprising the DQS composite score. Both of these limitations of the DQS may have affected the results ${ }^{(31)}$. In the categorical covariates (smoking status, alcohol consumption and physical activity), there was a varying degree of missing values. This could have affected the regression estimates. When analysing the individual food groups, suPAR varied significantly with fruit, vegetable and fat intake but not with fish intake. Fish intake within recommended dietary amounts is found to reduce CVD risk and inflammation in the general population ${ }^{(14,32,33)}$, and suPAR is a marker of inflammation and cardiovascular events ${ }^{(3,34)}$. The lack of variation in mean suPAR concentration between the categories of fish intake is therefore somewhat surprising but may be explained by the univariate nature of the analysis and the limitations of the DQS mentioned above.

Regarding endpoints, we found that the DQS was not associated with the risk of IHD or stroke or with the mortality when adjusted for sex, age and lifestyle factors; adjusting for suPAR did not change the results. SuPAR was found to associate strongly with mortality and weakly with stroke risk, when the total effect of a one-unit suPAR difference was examined. The 
(a)

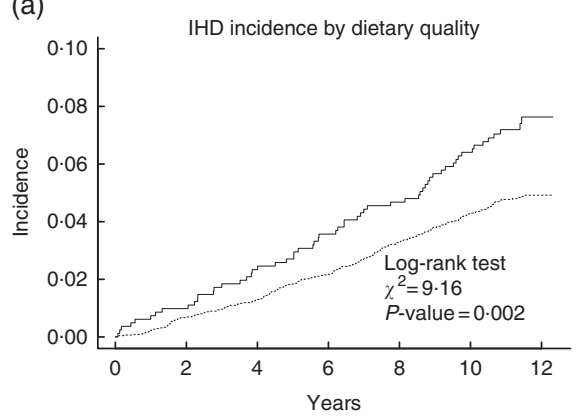

(b)

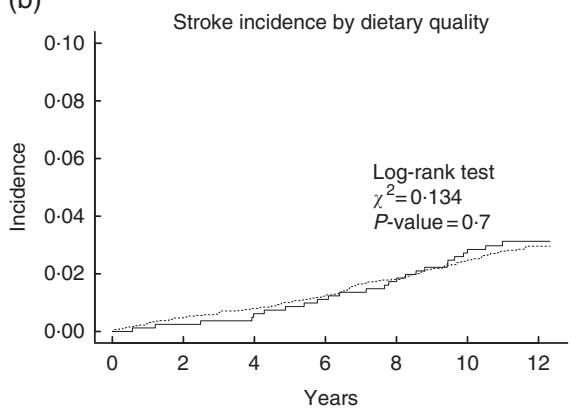

(c)

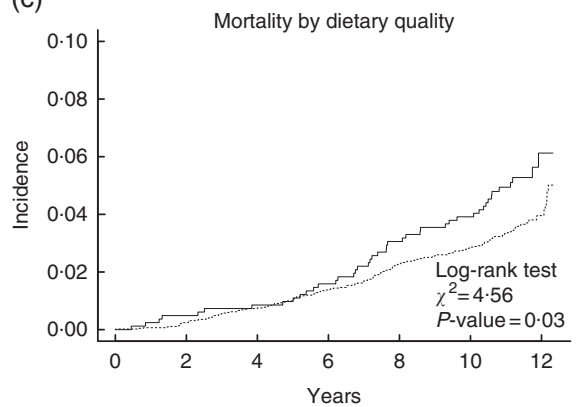

(d)

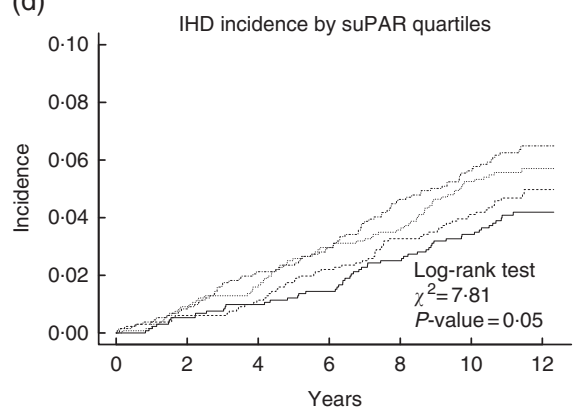

(e)

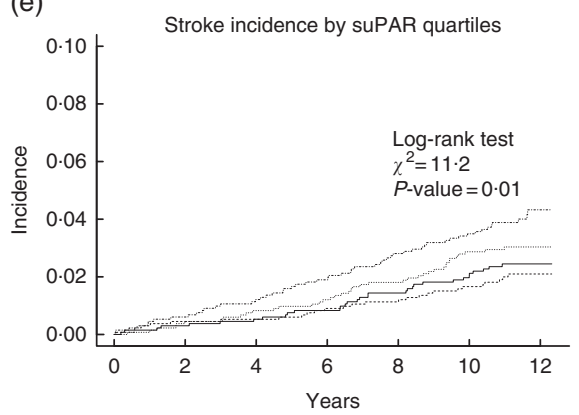

(f)

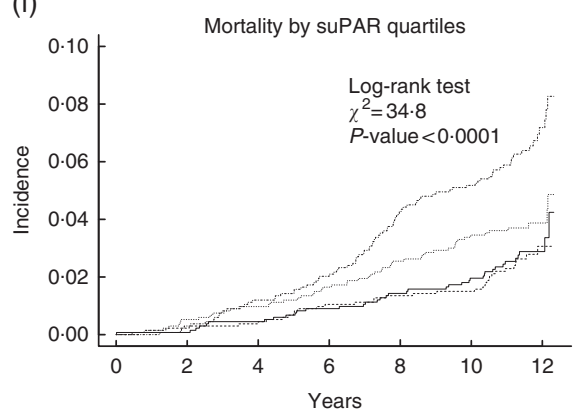

Fig. 4. Kaplan-Meier estimate curves showing differences in IHD-free probability, stroke-free probability and survival probability between an unhealthy diet (—-) and an intermediate or healthy diet $(----, a-c)$ and between sex- and age-specific ( $\leq 45$ years or $>45$ years) soluble urokinase plasminogen activator receptor (suPAR) quartiles $(\mathrm{d}-\mathrm{f})$ through the follow-up period of approximately 12 years. IHD-free and stroke-free probabilities are calculated, with death as a competing endpoint. Log-rank tests were performed and the $X^{2}$ values and $P$ values are listed by the corresponding graph. suPAR quartiles: Q1 ( - ): 0.65-2.99 ng/ml; Q2 $(----)$ : 2.52-3.64 ng/ml; Q3 (........): 3.04-4.59 $\mathrm{ng} / \mathrm{ml} ;$ Q4 (.-.-.): 3.79-21.2 ng/ml.

association between suPAR and stroke risk after adjustment for blood pressure, which is the most important risk factor for stroke ${ }^{(35)}$, is in agreement with another study showing suPAR as an independent predictor of ischaemic stroke ${ }^{(5)}$. However, since stroke was included as a composite endpoint in the present study, we were unable to test whether suPAR only explained the difference in the risk of ischaemic and not haemorrhagic stroke or whether suPAR also predicts haemorrhagic stroke independently of blood pressure. Association between suPAR and IHD risk has been shown earlier in a large population cohort ${ }^{(36)}$, and since suPAR is highly predictive of mortality $^{(3-7)}$, we speculate that the association between suPAR and IHD risk may be driven by high case fatality rates in historic cohorts ${ }^{(37-39)}$. Missing values regarding survival analyses generally seem to produce a slight underestimation of effect of diet on IHD risk and a slight overestimation of the effect of diet on stroke risk. The effect of suPAR on IHD and stroke and the estimates for all-cause mortality seems to be unaffected by the missing values. We also tested for interactions between age and DQS and age and suPAR, respectively. From the data presented in online Supplementary Table S3, it seems as though there generally is a tendency towards less importance of suPAR and more importance of dietary patterns in risk prediction in older individuals. The exception is IHD, where there seems to be less importance of diet with age. Dietary quality has previously been shown to predict mortality and CVD, but our results suggest that these endpoints are poorly predicted by the DQS. The DQS is, on the other hand, associated linearly with suPAR concentration, which in turn associates strongly with mortality. Thus, our study suggests that DQS may be associated with mortality, though this is only indicated through the association with suPAR concentration.

\section{Perspectives}

Results from this and other cohort studies have shown that suPAR is strongly associated with dietary quality, smoking 
habits and smoking cessation as well as other lifestyle factors $^{(8,9)}$. Considered together with the location of suPAR in the causal chain as represented in our directed acyclic graph, the before mentioned results make way to a new hypothesis: that suPAR can be seen as a marker of general health. A marker that sums up the influence of different predispositions, lifestyle factors and disease processes in a single mortality measure. Further, suPAR may reflect the combined health effect of all these factors including all possible interactions between them that the examination of the individual factors prevents us from quantifying.

\section{Limitations}

This study has certain limitations. It uses the cohort from the Inter99 study, which was not designed to show differences in suPAR or time to event between participants; therefore, associations may be underestimated. The cross-sectional design used to examine associations between diet and suPAR at baseline made us unable to determine whether a change in dietary habits causally affects the concentration of suPAR and in turn the risk of CVD or mortality. Likewise, we were unable to control for any changes in lifestyle or biomarkers through the 12 years of follow-up. Finally, the number of events with regard to incident stroke was low and results should therefore be cautiously interpreted and repeated in a larger study. This study was based on self-reported lifestyle data and may be affected by social desirability bias, misinterpretation of questions or mistakes in the reported answers. Furthermore, the categorisation of lifestyle habits based on the questionnaire used in the Inter99 study was limited in certain ways: we were not able to distinguish whether participants abstained from alcohol because of health-related reasons or as part of a healthy lifestyle; and we were not able to assess the energetic equivalents of exercise, as there was no detailed description of types of exercise for each individual. The DQS is based on a short FFQ of forty-eight items. This makes it a rough index of dietary habits and prevents inferences about the effect of dietary components. Furthermore, it may lead to misclassification bias with respect to the actual quality of the diet. To avoid such misclassification, the DQS was constructed according to the Danish national dietary recommendations and to index the intake of recommended food groups. Second, it was validated against a 198-item FFQ and the validation and performance of the DQS in risk prediction were evaluated against other dietary indexes including the healthy eating index. It was found that the DQS performed reasonably well in comparison with other scores and was well correlated with important measurements of a generally healthy diet: fibre, low intake of saturated fat, fruits, vegetables and fish $^{(22)}$. In addition, the short formulation of the FFQ might have reduced non-response and thus reduced the risk of introducing bias ${ }^{(40)}$. As we use the DQS in the same cohort it is developed in, we run the risk of autocorrelation of results. On the other hand, it is advised to use an index developed with the diet of the examined population in mind to best fit the actual dietary patterns of that population ${ }^{(41,42)}$; the DQS was the best fitted tool for use in a Danish adult population that we had available. This study also has several strengths, including the high number 
of participants and the high validity of the registries used to track endpoints.

\section{Conclusion}

We showed that suPAR was strongly associated with the DQS, both in univariate and in adjusted analyses, whereas cholesterol and BMI were not. The DQS was not associated with IHD risk, stroke risk or mortality, when adjusted for sex, age and other lifestyle factors. suPAR was strongly associated with mortality and, to a lesser degree, with stroke risk, but not with IHD risk, when adjusted for sex, age, lifestyle factors and other cardiovascular risk factors. Further studies are needed to confirm the found relationship between dietary quality and suPAR - preferably by using other scoring methods and different cohorts. Longitudinal studies are needed to investigate a causal link between suPAR and dietary quality over time and whether a change in suPAR concentration due to changes in dietary habits in turn affects disease risk.

\section{Acknowledgements}

We are grateful to Christian Thorball and Tomasz Pielak for measuring samples for suPAR and to Thomas Kallemose for statistical assistance.

P. B. S. T. has received a scholar grant from Herlev Hospital. None of the other authors has any funding to declare. The kits for measuring suPAR were kindly donated by ViroGates. ViroGates had no influence on the design of the study, on the interpretation of data or on the decision to publicise the data.

C. P. and U. T. were involved in the design and execution of the Inter99 study; P. B. S. T., T. H. H. and J. E.-O. formulated the research question; P. B. S. T., T. H. H. and S. L. performed statistical analysis; P. B. S. T., T. H. H. and J. E.-O. wrote the article; and all authors read and commented on the paper and had primary responsibility for final content.

J. E.-O. is co-founder of, and shareholder in, ViroGates A/S, Denmark. ViroGates has an exclusive right to use patents on suPAR that are owned by Copenhagen University Hospital Hvidovre and, on these, J. E.-O. is one of the inventors. The remaining authors have no conflicts of interest to report.

\section{Supplementary material}

For supplementary material/s referred to in this article, please visit https://doi.org/10.1017/S0007114518003720

\section{References}

1. Candore G, Caruso C, Jirillo E, et al. (2010) Low grade inflammation as a common pathogenetic denominator in agerelated diseases: novel drug targets for anti-ageing strategies and successful ageing achievement. Curr Pharm Des 16, 584-596.

2. Minihane AM, Vinoy S, Russell WR, et al. (2015) Low-grade inflammation, diet composition and health: current research evidence and its translation. Br J Nutr 114, 999-1012.
3. Thuno M, Macho B \& Eugen-Olsen J (2009) suPAR: the molecular crystal ball. Dis Markers 27, 157-172.

4. Eugen-Olsen J, Andersen O, Linneberg A, et al. (2010) Circulating soluble urokinase plasminogen activator receptor predicts cancer, cardiovascular disease, diabetes and mortality in the general population. J Intern Med 268, 296-308.

5. Persson M, Engstrom G, Bjorkbacka H, et al. (2012) Soluble urokinase plasminogen activator receptor in plasma is associated with incidence of CVD. Results from the Malmo Diet and Cancer Study. Atherosclerosis 220, 502-505.

6. Eugen-Olsen J \& Giamarellos-Bourboulis EJ (2015) suPAR: the unspecific marker for disease presence, severity and prognosis. Int J Antimicrob Agents 46, Suppl. 1, S33-S34.

7. Rasmussen LJ, Ladelund S, Haupt TH, et al. (2016) Soluble urokinase plasminogen activator receptor (suPAR) in acute care: a strong marker of disease presence and severity, readmission and mortality. A retrospective cohort study. Emerg Med J 33, 769-775.

8. Eugen-Olsen J, Ladelund S \& Sorensen LT (2016) Plasma suPAR is lowered by smoking cessation: a randomized controlled study. Eur J Clin Invest 46, 305-311.

9. Haupt TH, Kallemose T, Ladelund S, et al. (2014) Risk factors associated with serum levels of the inflammatory biomarker soluble urokinase plasminogen activator receptor in a general population. Biomark Insights 9, 91-100.

10. Akbaraly TN, Shipley MJ, Ferrie JE, et al. (2015) Long-term adherence to healthy dietary guidelines and chronic inflammation in the prospective Whitehall II study. Am J Med 128, 152-160.e154.

11. Pounis G, Bonaccio M, Di Castelnuovo A, et al. (2016) Polyphenol intake is associated with low-grade inflammation, using a novel data analysis from the Moli-sani study. Thromb Haemost 115, 344-352.

12. Roager HM, Vogt JK, Kristensen M, et al. (2017) Whole grain-rich diet reduces body weight and systemic low-grade inflammation without inducing major changes of the gut microbiome: a randomised cross-over trial. Gut $\mathbf{6 8}$, 83-93.

13. Boden S, Wennberg M, Van Guelpen B, et al. (2017) Dietary inflammatory index and risk of first myocardial infarction: a prospective population-based study. Nutr J 16, 21.

14. Sala-Vila A, Estruch R \& Ros E (2015) New insights into the role of nutrition in CVD prevention. Curr Cardiol Rep 17, 26.

15. Reidlinger DP, Darzi J, Hall WL, et al. (2015) How effective are current dietary guidelines for cardiovascular disease prevention in healthy middle-aged and older men and women? A randomized controlled trial. Am J Clin Nutr 101, 922-930.

16. Roswall N, Sandin S, Lof M, et al. (2015) Adherence to the healthy Nordic food index and total and cause-specific mortality among Swedish women. Eur J Epidemiol 30, 509-517.

17. Letois F, Mura T, Scali J, et al. (2016) Nutrition and mortality in the elderly over 10 years of follow-up: the Three-City study. $\mathrm{Br}$ J Nutr 116, 882-889.

18. Schulze MB \& Hoffmann K (2006) Methodological approaches to study dietary patterns in relation to risk of coronary heart disease and stroke. Br J Nutr 95, 860-869.

19. Jorgensen T, Borch-Johnsen K, Thomsen TF, et al. (2003) A randomized non-pharmacological intervention study for prevention of ischaemic heart disease: baseline results Inter99. Eur J Cardiovasc Prev Rehabil 10, 377-386.

20. Jorgensen T, Jacobsen RK, Toft U, et al. (2014) Effect of screening and lifestyle counselling on incidence of ischaemic heart disease in general population: Inter99 randomised trial. BMJ 348, g3617.

21. Pedersen CB (2011) The Danish civil registration system. Scand J Public Health 39, 22-25. 
22. Toft U, Kristoffersen LH, Lau C, et al. (2007) The dietary quality score: validation and association with cardiovascular risk factors: the Inter99 study. Eur J Clin Nutr 61, 270-278.

23. von Huth Smith L, Borch-Johnsen K \& Jorgensen T (2007) Commuting physical activity is favourably associated with biological risk factors for cardiovascular disease. Eur J Epidemiol 22, 771-779.

24. Lynge E, Sandegaard JL \& Rebolj M (2011) The Danish national patient register. Scand J Public Health 39, 30-33.

25. Helweg-Larsen K (2011) The Danish register of causes of death. Scand J Public Health 39, 26-29.

26. Friedewald WT, Levy RI \& Fredrickson DS (1972) Estimation of the concentration of low-density lipoprotein cholesterol in plasma, without use of the preparative ultracentrifuge. Clin Chem 18, 499-502.

27. Pearl J (1995) Causal diagrams for empirical research. Biometrika 82, 669-688.

28. Schisterman EF, Cole SR \& Platt RW (2009) Overadjustment bias and unnecessary adjustment in epidemiologic studies. Epidemiology 20, 488-495.

29. Escola-Gil JC, Julve J, Griffin BA, et al. (2015) HDL and lifestyle interventions. Handb Exp Pharmacol 224, 569-592.

30. Malik VS, Pan A, Willett WC, et al. (2013) Sugar-sweetened beverages and weight gain in children and adults: a systematic review and meta-analysis. Am J Clin Nutr 98, 1084-1102.

31. Willett WC, Howe GR \& Kushi LH (1997) Adjustment for total energy intake in epidemiologic studies. Am J Clin Nutr 65, 1220S-1228S; discussion 1229S-1231S.

32. Mozaffarian D, Lemaitre RN, King IB, et al. (2011) Circulating long-chain omega-3 fatty acids and incidence of congestive heart failure in older adults: the cardiovascular health study: a cohort study. Ann Intern Med 155, 160-170.

33. Robinson LE \& Mazurak VC (2013) $n-3$ polyunsaturated fatty acids: relationship to inflammation in healthy adults and adults exhibiting features of metabolic syndrome. Lipids 48, 319-332.
34. Hodges GW, Bang CN, Wachtell K, et al. (2015) suPAR: a new biomarker for cardiovascular disease? Can J Cardiol 31, 1293-1302.

35. Wolf PA, D'Agostino RB, Belanger AJ, et al. (1991) Probability of stroke: a risk profile from the Framingham study. Stroke $\mathbf{2 2}$, 312-318.

36. Persson M, Ostling G, Smith G, et al. (2014) Soluble urokinase plasminogen activator receptor: a risk factor for carotid plaque, stroke, and coronary artery disease. Stroke $\mathbf{4 5}$, $18-23$.

37. Buch P, Rasmussen S, Gislason GH, et al. (2007) Temporal decline in the prognostic impact of a recurrent acute myocardial infarction 1985 to 2002. Heart 93, 210-215.

38. Frost L, Andersen LV, Vestergaard P, et al. (2007) Trend in mortality after stroke with atrial fibrillation. Am J Med 120, $47-53$.

39. Schmidt M, Ulrichsen SP, Pedersen L, et al. (2016) Thirty-year trends in heart failure hospitalization and mortality rates and the prognostic impact of co-morbidity: a Danish nationwide cohort study. Eur J Heart Fail 18, 490-499.

40. Cleghorn C, Harrison RA, Ransley JK et al. (2016) Can a dietary quality score derived from a short-form FFQ assess dietary quality in UK adult population surveys? Public Health Nutr 19, 2915-2923.

41. Cade J, Thompson R, Burley V, et al. (2002) Development, validation and utilisation of food-frequency questionnaires - a reviewPublic Health Nutr 5, 567-587.

42. Yokoyama Y, Takachi R, Ishihara J, et al. (2016) Validity of short and long self-administered food frequency questionnaires in ranking dietary intake in middle-aged and elderly Japanese in the Japan Public Health Center-Based Prospective Study for the Next Generation (JPHC-NEXT) protocol area. J Epidemiol 26, 420-432.

( )

